\title{
RANK NUMBERS FOR BENT LADDERS
}

\author{
Peter Richter ${ }^{1}$, Emily Leven ${ }^{2}$ \\ Anh Tran ${ }^{3}$, Bryan EK ${ }^{4}$, Jobby JaCoB ${ }^{4}$ \\ AND \\ DARREN A. NARAYAN ${ }^{4}$ \\ ${ }^{1}$ Department of Mathematics, University of Rochester \\ Rochester, NY 14642-0002, USA \\ ${ }^{2}$ Department of Mathematics, University of California \\ San Diego, La Jolla, California, 92093-0112, USA \\ 3 Department of Mathematics, Temple University \\ Philadelphia PA 19122, USA \\ ${ }^{4}$ School of Mathematical Sciences \\ Rochester Institute of Technology \\ Rochester, NY 14623, USA \\ e-mail: prichter@u.rochester.edu \\ esergel07@gmail.com \\ anh.van.tran@temple.edu \\ bte1759@rit.edu \\ jxjsma@rit.edu \\ dansma@rit.edu
}

\begin{abstract}
A ranking on a graph is an assignment of positive integers to its vertices such that any path between two vertices with the same label contains a vertex with a larger label. The rank number of a graph is the fewest number of labels that can be used in a ranking. The rank number of a graph is known for many families, including the ladder graph $P_{2} \times P_{n}$. We consider how "bending" a ladder affects the rank number. We prove that in certain cases the rank number does not change, and in others the rank number differs by only 1 . We investigate the rank number of a ladder with an arbitrary number of bends.
\end{abstract}

Keywords: graph colorings, rankings of graphs, rank number, Cartesian product of graphs, ladder graph, bent ladder graph.

2010 Mathematics Subject Classification: 05C78, 05C15, $05 \mathrm{C} 76$. 


\section{REFERENCES}

[1] H. Alpert, Rank numbers of grid graphs, Discrete Math. 310 (2010) 3324-3333. doi:10.1016/j.disc.2010.07.022

[2] H.L. Bodlaender, J.S. Deogun, K. Jansen, T. Kloks, D. Kratsch, H. Müller and Zs. Tuza, Rankings of graphs, SIAM J. Discrete Math. 11 (1998) 168-181. doi:10.1137/S0895480195282550

[3] E. Bruoth and M. Horňák, Online-ranking numbers for cycles and paths, Discuss. Math. Graph Theory 19 (1999) 175-197. doi:10.7151/dmgt.1094

[4] C.-W. Chang, D. Kuo and H-C. Lin, Ranking numbers of graphs, Inform. Process. Lett. 110 (2010) 711-716. doi:10.1016/j.ipl.2010.05.025

[5] D. Dereniowski, Rank coloring of graphs, in: Graph Colorings, M. Kubale (Ed.), Contemp. Math. AMS 352 (2004) 79-93. doi:10.1090/conm/352/06

[6] J. Ghoshal, R. Laskar, and D. Pillone, Minimal rankings, Networks 28 (1996) 45-53. doi:10.1002/(SICI)1097-0037(199608)28:1〈45::AID-NET6〉3.0.CO;2-D

[7] A.V. Iyer, H.D. Ratliff and G. Vijayan, Optimal node ranking of trees, Inform. Process. Lett. 28 (1988) 225-229. doi:10.1016/0020-0190(88)90194-9

[8] R.E. Jamison, Coloring parameters associated with the rankings of graphs, Congr. Numer. 164 (2003) 111-127.

[9] M. Katchalski, W. McCuaig and S. Seager, Ordered colourings, Discrete Math. 142 (1995) 141-154. doi:10.1016/0012-365X(93)E0216-Q

[10] T. Kloks, H. Müller and C.K. Wong, Vertex ranking of asteroidal triple-free graphs, Inform. Process. Lett. 68 (1998) 201-206. doi:10.1016/S0020-0190(98)00162-8

[11] C.E. Leiserson, Area efficient graph layouts for VLSI, Proc. 21st Ann. IEEE Symposium, FOCS (1980) 270-281.

[12] S. Novotny, J. Ortiz, and D.A. Narayan, Minimal k-rankings and the rank number of $P_{n}^{2}$, Inform. Process. Lett. 109 (2009) 193-198. doi:10.1016/j.ipl.2008.10.004

[13] J. Ortiz, H. King, A. Zemke and D.A. Narayan, Minimal k-rankings for prism graphs, Involve 3 (2010) 183-190. doi:10.2140/involve.2010.3.183

[14] A. Sen, H. Deng and S. Guha, On a graph partition problem with application to VLSI Layout, Inform. Process. Lett. 43 (1992) 87-94. doi:10.1016/0020-0190(92)90017-P 
Received 11 August 2011

Revised 4 March 2013

Accepted 28 March 2013 